PROCEEDINGS OF THE AMERICAN MATHEMATICAL SOCIETY

Volume 124, Number 3, March 1996

\title{
THE ALGEBRA OF ALMOST PERIODIC FUNCTIONS HAS INFINITE TOPOLOGICAL STABLE RANK
}

\author{
FERNANDO DANIEL SUÁREZ \\ (Communicated by Dale Alspach)
}

\begin{abstract}
We show that if $A$ is the uniform algebra of almost periodic functions, then the set $U_{n}(A)=\left\{\left(a_{1}, \ldots, a_{n}\right) \in A^{n}: \sum_{1 \leq j \leq n} A a_{j}=A\right\}$ cannot be dense in $A^{n}$ for any positive integer $n$.
\end{abstract}

By a Banach algebra we mean a commutative complex Banach algebra with unit. Let $B$ be a Banach algebra and $n$ be a positive integer. The set of unimodulars of $B$ is $U_{n}(B)=\left\{\left(b_{1}, \ldots, b_{n}\right) \in B^{n}: \sum_{1<j \leq n} B b_{j}=B\right\}$, and the topological stable rank of $B(\operatorname{tsr} B)$ is the minimum positive integer $n$ such that $U_{n}(B)$ is dense in $B^{n}$. We write $\operatorname{tsr} B=\infty$ if such $n$ does not exist. Since its introduction by Rieffel $[5]$ this concept has been very successful in studying the topological $K$-theory and some spectral properties of Banach algebras.

For a Banach algebra $B$, denote by $B^{*}$ its dual space provided with the weak* topology. The maximal ideal space of $B$ is the compact Hausdorff space

$$
X(B)=\left\{\varphi \in B^{*}: \varphi \text { is multiplicative, } \varphi \neq 0\right\} .
$$

We denote by $C(X(B))$ the uniform algebra of continuous complex-valued functions on $X(B)$. The Gelfand transform ^ ${ }^{\wedge} B \rightarrow C(X(B))$, defined by $\hat{b}(\varphi)=\varphi(b)$, is a Banach algebras morphism. So, for every positive integer $n$ and every $b \in B^{n}$ the Gelfand transform induces a continuous function $\hat{b}: X(B) \rightarrow \mathbb{C}^{n}$. It is an easy exercise to prove that $b \in U_{n}(B)$ if and only if $0 \notin \hat{b}(X(B))$.

The algebra $A$ of almost periodic functions is the uniform algebra on $\mathbb{R}$ generated by the functions

$$
g(t)=\sum_{1 \leq k \leq n} c_{k} e^{i \lambda_{k} t} \quad(t \in \mathbb{R}),
$$

where $n$ is a positive integer, $c_{k} \in \mathbb{C}$ and $\lambda_{k} \in \mathbb{R}$ for $k=1, \ldots, n$. See [1, pp. 16 and $164]$ for general background about this algebra. The space $X(A)$ is the so-called Bohr compactification of $\mathbb{R}$, and it is well known that $\mathbb{R}$ is dense in $X(A)$ [1]. So, a necessary and sufficient condition for $\left(a_{1}, \ldots, a_{n}\right) \in A^{n}$ to be unimodular is that $\left|a_{1}(t)\right|^{2}+\cdots+\left|a_{n}(t)\right|^{2} \geq \delta>0$ for all $t \in \mathbb{R}$. As said in the abstract, the purpose of this paper is to show that $\operatorname{tsr} A=\infty$. This problem was posed by I. Spitkovsky

Received by the editors July 12, 1994 and, in revised form, September 22, 1994.

1991 Mathematics Subject Classification. Primary 46J10.

Key words and phrases. Almost periodic functions, unimodulars, topological stable rank.

The author is a Fellow of the John Simon Guggenheim Memorial Foundation.

(C)1996 American Mathematical Society 
in a lecture where D. Sarason was present; I am grateful to him for communicating the problem to me.

We begin by establishing some conventions. If $X$ is a compact Hausdorff space and $Y$ is a metric space, then $C(X, Y)$ denotes the space of continuous functions from $X$ into $Y$ with the supremum metric. We simply write $C(X)^{n}$ when $Y=$ $\mathbb{C}^{n}$. By a polynomial in $z_{1}, \ldots, z_{n}$ we mean a finite complex linear combination of $z_{1}^{p_{1}} \cdots z_{n}^{p_{n}}$, where $p_{j} \in \mathbb{Z}$ (the integer group). A $\mathbb{C}^{n}$-valued polynomial means a function with values in $\mathbb{C}^{n}$ where each coordinate is a polynomial. It is clear that every function $g$ as in (1) can be written as a polynomial $f\left(e^{i \lambda_{1} t}, \ldots, e^{i \lambda_{m} t}\right)$, where $\lambda_{1}, \ldots, \lambda_{m}$ are linearly independent over $\mathbb{Z}$. Therefore Kronecker's theorem $\left[2\right.$, Theorem 443] implies that the set $\left\{\left(e^{i \lambda_{1} t}, \ldots, e^{i \lambda_{m} t}\right): t \in \mathbb{R}\right\}$ is dense in the $m$-dimensional torus $\mathbb{T}^{m}$, and we can identify $g$ with a polynomial on the set $\mathbb{T}^{m}$. It is well known that the set of polynomials on $\mathbb{T}^{m}$ is dense in the algebra $C\left(\mathbb{T}^{m}\right)$. For $s$ a positive integer we define $\nu_{s}: \mathbb{T}^{m} \rightarrow \mathbb{T}^{m}$ by $\nu_{s}\left(\omega_{1}, \ldots, \omega_{m}\right)=\left(\omega_{1}^{s}, \ldots, \omega_{m}^{s}\right)$. In the sequel \|\| denotes the euclidean norm in $\mathbb{C}^{n}$.

Lemma 1. Let $f$ be $a \mathbb{C}^{n}$-valued polynomial on $e^{i \lambda_{1} t}, \ldots, e^{i \lambda_{m} t}$, where $\lambda_{1}, \ldots, \lambda_{m} \in$ $\mathbb{R}$ are linearly independent over $\mathbb{Z}$. Then $f \in \overline{U_{n}(A)}$ if and only if there is a sequence of positive integers $\left\{s_{j}\right\}$ such that

$$
\operatorname{dist}\left(f \circ \nu_{s_{j}}, U_{n}\left(C\left(\mathbb{T}^{m}\right)\right)\right) \rightarrow 0
$$

when $j \rightarrow \infty$.

Proof. If (2) holds, then for any $\varepsilon>0$ there is an integer $s>0$ and a polynomial $F: \mathbb{T}^{m} \rightarrow \mathbb{C}_{*}^{n}=\mathbb{C}^{n} \backslash\{0\}$ so that

$$
\sup _{\omega \in \mathbb{T}^{m}}\left\|F(\omega)-f \circ \nu_{s}(\omega)\right\|<\varepsilon .
$$

Then for every $t \in \mathbb{R}$,

$$
\left\|F\left(e^{i \frac{\lambda_{1}}{s} t}, \ldots, e^{i \frac{\lambda_{m}}{s} t}\right)-f\left(e^{i \lambda_{1} t}, \ldots, e^{i \lambda_{m} t}\right)\right\|<\varepsilon .
$$

Since $\varepsilon$ is arbitrary, $f \in \overline{U_{n}(A)}$. On the other hand, if $f \in \overline{U_{n}(A)}$, then for $\varepsilon>0$ there are $\mu_{1}, \ldots, \mu_{k} \in \mathbb{R}$ and a $\mathbb{C}_{*}^{n}$-valued polynomial $F$ on $e^{i \lambda_{1} t}, \ldots, e^{i \lambda_{m} t}, e^{i \mu_{1} t}, \ldots$, $e^{i \mu_{k} t}$ such that

$$
\sup _{t \in \mathbb{R}}\left\|F\left(e^{i \lambda_{1} t}, \ldots, e^{i \lambda_{m} t}, e^{i \mu_{1} t}, \ldots, e^{i \mu_{k} t}\right)-f\left(e^{i \lambda_{1} t}, \ldots, e^{i \lambda_{m} t}\right)\right\|<\varepsilon .
$$

We can reduce the number of variables in the writing of $F$ by the following process. If for every linear combination

$$
p_{1} \lambda_{1}+\cdots+p_{m} \lambda_{m}+q_{1} \mu_{1}+\cdots+q_{k} \mu_{k}=0
$$

with $p_{j}, q_{j} \in \mathbb{Z}$ we have that $q_{k}=0$, then we keep $\mu_{k}$ and we repeat the process with $\lambda_{1}, \ldots, \mu_{k-1}$. If there is a combination (3) with $q_{k} \neq 0$, then we eliminate $\mu_{k}$ and repeat the process with $\lambda_{1} /\left|q_{k}\right|, \ldots, \mu_{k-1} /\left|q_{k}\right|$. After finite steps we obtain a positive integer $s$ and $\lambda_{1} / s, \ldots, \lambda_{m} / s, \mu_{1}^{\prime}, \ldots, \mu_{l}^{\prime} \in \mathbb{R}$ (with $l \leq k$ ) linearly independent over $\mathbb{Z}$, so that $F$ can be written as a $\mathbb{C}_{*}^{n}$-valued polynomial in the correspondent exponentials. Consequently,

$$
\sup _{t \in \mathbb{R}}\left\|F\left(e^{i \lambda_{1} t}, \ldots, e^{i \lambda_{m} t}, e^{i s \mu_{1}^{\prime} t}, \ldots, e^{i s \mu_{l}^{\prime} t}\right)-f\left(e^{i \lambda_{1} s t}, \ldots, e^{i \lambda_{m} s t}\right)\right\|<\varepsilon .
$$

Define $\widetilde{F}: \mathbb{T}^{m} \rightarrow \mathbb{C}_{*}^{n}$ by $\widetilde{F}\left(\omega_{1}, \ldots, \omega_{m}\right)=F\left(\omega_{1}, \ldots, \omega_{m}, 1, \ldots, 1\right)$. Then $\widetilde{F} \in$ $U_{n}\left(C\left(\mathbb{T}^{m}\right)\right)$ and $\sup _{\mathbb{T}^{m}}\left\|\widetilde{F}-f \circ \nu_{s}\right\|<\varepsilon$, as claimed. 
Our next lemma requires a classical result of topology due to Borsuk [4, Theorem III.3]. Let $X$ be a compact Hausdorff space and $Z \subset X$ closed. If $f, g \in C\left(Z, \mathbb{C}_{*}^{n}\right)$ are homotopic and there is an extension $G \in C\left(X, \mathbb{C}_{*}^{n}\right)$ of $g$, then there is also an extension $F \in C\left(X, \mathbb{C}_{*}^{n}\right)$ of $f$. For $f \in C(X)^{n}$ put

$$
E(f)=\inf \left\{\delta \geq\left. 0 f\right|_{(\|f\|=\delta)} \text { admits an extension } F X \rightarrow \mathbb{C}_{*}^{n}\right\} .
$$

Suppose that $\delta>E(f)$. Then by definition of $E(f)$ there are $\delta_{0}<\delta$ and an extension $F_{0} X \rightarrow \mathbb{C}_{*}^{n}$ of $\left.f\right|_{\left(\|f\|=\delta_{0}\right)}$. Henceforth, the function $F(x)$ defined as $F_{0}(x)$ when $\|f(x)\| \leq \delta_{0}$ and $f(x)$ when $\|f(x)\|>\delta_{0}$ is an extension of $\left.f\right|_{(\|f\|=\delta)}$ from $X$ into $\mathbb{C}_{*}^{n}$.

Lemma 2. $E(f) \leq \operatorname{dist}\left(f, U_{n}(C(X))\right) \leq 2 E(f)$.

Proof. Let $\delta>E(f)$ and let $F X \rightarrow \mathbb{C}_{*}^{n}$ be an extension of $\left.f\right|_{(\|f\|=\delta)}$. Define

$$
\mathcal{F}(x)= \begin{cases}f(x) & \text { if }\|f(x)\| \geq \delta \\ \delta \frac{F(x)}{\|F(x)\|} & \text { if }\|f(x)\| \leq \delta .\end{cases}
$$

Therefore $\mathcal{F} \in U_{n}(C(X))$ and its distance to $f$ is

$$
\sup _{\|f(x)\| \leq \delta}\|\delta F(x) /\| F(x)\|-f(x)\| \leq 2 \delta .
$$

For the other inequality put $d=\operatorname{dist}\left(f, U_{n}(C(X))\right)$. If $\delta>d$, then there is $G \in$ $C\left(X, \mathbb{C}_{*}^{n}\right)$ such that $\sup _{X}\|f-G\|<\delta$. Hence, $f+\left.t(G-f)\right|_{(\|f\|=\delta)}(0 \leq t \leq 1)$ is a homotopy in $C\left((\|f\|=\delta), \mathbb{C}_{*}^{n}\right)$ between the restrictions of $f$ and $G$ to $(\|f\|=\delta)$. Consequently Borsuk's theorem assures that there is an extension $F \in C\left(X, \mathbb{C}_{*}^{n}\right)$ of $\left.f\right|_{(\|f\|=\delta)}$. That is, $E(f) \leq \delta$. Since $\delta>d$ is arbitrary, the lemma follows.

Theorem 3. The topological stable rank of $A$ is infinite.

Proof. Let $n$ be a positive integer. Since the polynomials on $\mathbb{T}^{2 n}$ are dense in $C\left(\mathbb{T}^{2 n}\right)$, Lemmas 1 and 2 imply that if there is an $f \in C\left(\mathbb{T}^{2 n}\right)^{n}$ such that $E\left(f \circ \nu_{s}\right) \geq$ 1 for all positive integers $s$, then $\operatorname{tsr} A \geq n$.

Let $\mathbb{T}_{s}^{2 n}$ be the $2 n$-times cartesian product of $\left\{e^{i \theta}|\theta| \leq \pi / 2 s\right\}$. Then the map $\tilde{\nu}_{s}=\left.\nu_{s}\right|_{\partial \mathbb{T}_{s}^{2 n}}$ is a homeomorphism from $\partial \mathbb{T}_{s}^{2 n}$ onto $\partial \mathbb{T}_{1}^{2 n}$.

The set $\mathbb{T}_{1}^{2 n}$ is a product of $2 n$ closed arc-intervals. Since each arc-interval is homeomorphic to $I=[0,1]$, then $\mathbb{T}_{1}^{2 n}$ is homeomorphic to $I^{2 n}$, and therefore to $B_{n}=\left\{z \in \mathbb{C}^{n}\|z\| \leq 1\right\}$. Let $\varphi \mathbb{T}_{1}^{2 n} \rightarrow B_{n}$ be an onto homeomorphism, and take any $f \in C\left(\mathbb{T}^{2 n}, B_{n}\right)$ so that $\left.f\right|_{\mathbb{T}_{1}^{2 n}}=\varphi$. Clearly

$$
f\left(\nu_{s}\left(\partial \mathbb{T}_{2}^{2 n}\right)\right)=f\left(\partial \mathbb{T}_{1}^{2 n}\right)=\varphi\left(\partial \mathbb{T}_{1}^{2 n}\right)=\partial B_{n}
$$

In other words, $\partial \mathbb{T}_{s}^{2 n}$ is contained in $\left\{\omega \in \mathbb{T}^{2 n}\left\|\left(f \circ \nu_{s}\right)(\omega)\right\|=1\right\}$. So, if $E\left(f \circ \nu_{s}\right)<1$, then there exists some extension $F_{s} \in C\left(\mathbb{T}_{s}^{2 n}, \mathbb{C}_{*}^{n}\right)$ of $\left.f \circ \nu_{s}\right|_{\partial \mathbb{T}_{s}^{2 n}}=\varphi \circ \tilde{\nu}_{s}$, and since $\mathbb{T}_{s}^{2 n}$ is a contractible space, this only happens if $\varphi \circ \tilde{\nu}_{s}$ is homotopic to some constant function in the space $C\left(\partial \mathbb{T}_{s}^{2 n}, \partial B_{n}\right)$. Put $\varphi_{-1}=\left(\left.\varphi\right|_{\partial \mathbb{T}_{1}^{2 n}}\right)^{-1}$ and consider the following string of mappings:

$$
\partial B_{n} \stackrel{\varphi_{-1}}{\longrightarrow} \partial \mathbb{T}_{1}^{2 n} \stackrel{\tilde{\nu}_{s}^{-1}}{\longrightarrow} \partial \mathbb{T}_{s}^{2 n} \stackrel{\tilde{\nu}_{s}}{\longrightarrow} \partial \mathbb{T}_{1}^{2 n} \stackrel{\varphi}{\longrightarrow} \partial B_{n} .
$$

It is immediate that $\varphi \circ \tilde{\nu}_{s} \circ \tilde{\nu}_{s}^{-1} \circ \varphi_{-1}=\mathrm{id}_{\partial B_{n}}$. Therefore, if $\varphi \circ \tilde{\nu}_{s}$ is null-homotopic in $C\left(\partial \mathbb{T}_{s}^{2 n}, \partial B_{n}\right)$, then so is $\operatorname{id}_{\partial B_{n}}$ in $C\left(\partial B_{n}, \partial B_{n}\right)$, which is clearly false. Thus $E\left(f \circ \nu_{s}\right) \geq 1$. 
The Bass stable rank of a Banach algebra $B$ (bsr $B$ ) is the minimum positive integer $n$ with the following property. For every $\left(b_{1}, \ldots, b_{n+1}\right) \in U_{n+1}(B)$ there are $c_{1}, \ldots, c_{n} \in B$ such that $\left(b_{1}+c_{1} b_{n+1}, \ldots, b_{n}+c_{n} b_{n+1}\right) \in U_{n}(B)$. We put bsr $B=\infty$ if there is no such $n$. This notion originates in algebraic $K$-theory and it is the direct antecessor of the topological stable rank.

It is interesting to notice that although the Bass stable rank is a purely algebraic invariant, it coincides with the topological stable rank in the special case of $C^{*}$ algebras (see [3]). Since the algebra $A$ is a $C^{*}$-algebra, Theorem 3 also says that $\operatorname{bsr} A=\infty$.

\section{REFERENCES}

1. T. W. Gamelin, Uniform algebras, Prentice-Hall, Englewood Cliffs, NJ, 1969. MR 53:14137

2. G. H. Hardy and E. M. Wright, An introduction to the theory of numbers, Clarendon Press, Oxford, 1979. MR 81i:10002

3. R. H. Herman and L. N. Vaserstein, The stable range of $C^{*}$-algebras, Invent. Math. 77 (1984), 553-555. MR 86a: 46074

4. J. Nagata, Modern Dimension Theory, revised and extended version, Heldermann Verlag, Berlin, 1983. MR 84h:54033

5. M. Rieffel, Dimension and stable rank in the $K$-theory of $C^{*}$-algebra, Proc. London Math. Soc. 46 (1983), 301-333. MR 84g:46085

Department of Mathematics, University of California, Berkeley, California 94720

Current address: Instituto Argentino de Matemática, Vio monte $1636-1^{\circ}$ Cpo.- $1^{\circ}$ Piso, 1055

Buenos Aires, Argentina 